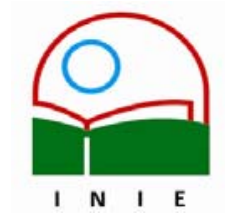

Universidad de Costa Rica

Facultad de Educación

Instituto de Investigación en Educación

ACTUALDADES INVESTIG ATIVASEN EDUCACION

\title{
EDUCACIÓN DE LA EXPRESIÓN DE LA SEXUALIDAD Y LA INTELIGENCIA EMOCIONAL EN NIÑAS, NIÑOS Y ADOLESCENTES CON DERECHOS
}

\author{
EDUCATION ON THE EXPRESSION OF SEXUALITY AND EMOTIONAL INTELLIGENCE \\ FOR GIRLS, BOYS AND TEENAGERS ENTITLED WITH RIGHTS
}

\author{
Sonia Carballo Vargas ${ }^{1}$ \\ "La Educación es sin duda el más humano y \\ humanizador de los empeños humanos" \\ Fernando Savater
}

\begin{abstract}
Resumen: En el presente artículo se integra la educación de la inteligencia emocional y la educación de la expresión de la sexualidad humana tomando como principal base los planteamientos de H. Gardner, D. Goleman y los aportes de la experiencia de la investigadora como educadora sexual, en la Universidad de Costa Rica y en el sistema educativo costarricense. A manera de conclusión se concretan lineamientos para educar en niños, niñas y adolescentes la expresión de la sexualidad en forma inteligente cognitiva y emocionalmente.
\end{abstract}

Palabras clave: EDUCACIÓN, INTELIGENCIA EMOCIONAL, EXPRESIÓN DE LA SEXUALIDAD, PERSONAS CON DERECHOS

\begin{abstract}
In the present article, the author brings together the education on emotional intelligence and the education on the expression of human sexuality; using the statements of $\mathrm{H}$. Gardner, D. Goleman and the contributions of the researcher's experience as a sexual educator in the University of Costa Rica and in the Costa Rican education system as her main foundation. In the conclusion, the article defines the guidelines for teaching emotionally and cognitively an intelligent expression of sexuality to boys, girls and teenagers.
\end{abstract}

Keywords: EDUCATION, EMOTIONAL INTELIGENCE, EXPRESSION OF SEXUALITY, PEOPLE ENTITLED WITH RIGHTS

\footnotetext{
${ }^{1}$ Profesora Catedrática de la Universidad de Costa Rica, Investigadora del Instituto de Investigaciones en Educación, Directora de la Escuela de Formación Docente de la Facultad de Educación de la Universidad de Costa Rica y Coordinadora de la Comisión Compartida en la Enseñanza de la Psicología. Tiene publicaciones en Revistas de Educación de la Universidad de Costa Rica y la Universidad Nacional.
}

Correo electrónico: fordocente@cariari.ucr.ac.cr

Artículo recibido: 19 de junio, 2006

Aprobado: 11 de noviembre, 2006 


\section{Introducción}

Esta Integración de la educación de la inteligencia emocional y su trascendencia al educar a niños, a niñas y a adolescentes con derechos, en la educación de la expresión de su sexualidad se contextualizan dentro de los planteamientos de dos autores: Howard Gardner (1993) y Daniel Goleman (1995), (2003).

Gardner (1993) en su teoría de las múltiples inteligencias fundamenta una serie de talentos que tiene el ser humano, y con una visión integral del mismo presenta modos para adquirir y expresar lo talentos a través del lenguaje, del análisis lógico matemático de la representación especial, del pensamiento musical, del uso del cuerpo para resolver problemas y hacer cosas, de una comprensión de la persona misma y de una comprensión de los demás. Este amplio espectro de habilidades las ubica el autor en siete categorías: inteligencia lingüística, inteligencia lógico matemática, inteligencia espacial, inteligencia física y cinestésica, inteligencia musical, inteligencia intrapersonal e inteligencia interpersonal. Sin embargo, no descarta que la persona posea otras inteligencias, como la inteligencia espiritual, entendida ésta, como la capacidad para vivir orientada por un sistema de principios éticos.

El crecimiento y decrecimiento de cada una de estas inteligencias depende básicamente de las acciones recíprocas - transacciones - entre la herencia biológica, prácticas de crianza empleadas en la educación y el contexto histórico cultural dentro del cual vive la persona. Las inteligencias funcionan juntas de manera compleja dentro de cada ser humano y el desarrollo óptimo, en una o varias de ellas es el resultado de relación entre las transacciones señaladas.

Goleman (1995), impacta no sólo con los planteamientos que hace en el libro "Inteligencia Emocional", sino también, con las investigaciones y las experiencias que presenta para reflexionar sobre cómo influyen en el desarrollo humano las habilidades propias de las inteligencias intrapersonales e interpersonales, planteadas por Gardner, a las cuales él se refiere - en conjunto - como inteligencia emocional.

$\mathrm{Ni}$ el intelecto, ni la inteligencia emocional, pueden por sí solos actuar armónicamente. Cuando las personas conocen e interpretan con eficacia sus habilidades emocionales, se proyectan con mayor claridad hacia los sentimientos de los otros y contribuyen a dar satisfacción a sus vidas y a dominar hábitos mentales que favorecen su propia productividad. 
Son personas con una inteligencia emocional elevada, que se sienten bien consigo mismas, con las demás y con el universo que les rodea. Además, son socialmente equilibradas, solidarias, alegres y comprometidas en sus relaciones interpersonales (Goleman, 1995). Estas personas están facultadas para conocer y mejorar sus talentos e incapacidades.

Es por eso, que la humanidad necesita que los niños, las niñas y los y las adolescentes sean educados y educadas con prácticas de crianza inteligentes emocionalmente. Esto se logra cuando quienes conviven con ellos y ellas afrontan el reto de cultivar las habilidades de la inteligencia emocional en ellos mismos y en ellas mismas y en los niños, las niñas y los y las adolescentes, en forma sistemática.

De acuerdo con Goleman (1995) las habilidades de la inteligencia emocional se concretan en:

- Autoconocimiento: Es el conjunto de habilidades que permiten tomar conciencia de las fortalezas y debilidades que cada quién tiene; es la conciencia de lo que estoy sintiendo y haciendo y de su pertinencia en el contexto histórico-cultural; es la conciencia de mis derechos y deberes.

- Autodominio: Son las habilidades que en su conjunto permiten el equilibrio consciente y voluntario de las emociones, que permite que ellas fluyan en forma eficiente y pertinente y que se apliquen de manera oportuna y con la intensidad requerida en la situación correspondiente.

- Automotivación: Su estrecha relación con la emoción conduce al logro de las metas que se desean, hacia las cuales se enrumba la persona con todo su empeño, con toda su atención y con todo su entusiasmo.

- Empatía: Entendida como el conjunto de habilidades que le permiten al ser humano ponerse en el lugar del otro, sin dejar de ser él mismo (escucho, atiendo, expreso y reconozco en él su sentir para ayudarle sin evaluar y sin hacer juicios de valor).

- Relaciones sociales: Este conjunto de habilidades contribuyen a la convivencia cotidiana permitiendo manejar un lenguaje emocional que permita la comunicación, la 
negociación ante diversos retos, el respeto a la diversidad, el poder diferir y resolver los conflictos mediante el diálogo.

Las emociones están en cada ser humano desde la concepción y por ser la "sal" de la vida, hay que emplear prácticas de crianza que permitan educar el conjunto de habilidades emocionales necesarias para no permitir que las emociones se desborden, ni que se repriman. Para que el poder de la inteligencia emocional actúe hay que conocer, identificar y administrar nuestras emociones.

La autora de este artículo considera relevante concebir la educación de la expresión de la sexualidad fundamentada y orientada por las características propias de la inteligencia emocional y desde esta perspectiva se integran planteamientos y se construyen posiciones.

\section{Expresión de la sexualidad Humana e Inteligencia Emocional}

Cuando las necesidades básicas del niño y de la niña, durante sus primeros años de vida no son atendidas, a ellos y a ellas les cuesta desarrollar las habilidades emocionales de autoconocimiento, automotivación, autocontrol, empatía y relaciones sociales. Cuando no se orienta a la persona en los primeros años de vida, para que se autocontrole y autorregule su comportamiento, aumentan las posibilidades de que al crecer sus habilidades de autoconocimiento y de relaciones humanas no se encuentren fortalecidas para la convivencia consigo misma y con las demás personas y esto repercute en la expresión de su sexualidad.

Cuando a las personas adultas les corresponde asumir el papel de educadores, su propia educación se convierte en un derecho ineludible. Concretamente, educar la expresión de la sexualidad de los niños, niñas y adolescentes conscientes de que se hará de una forma inteligente cognitiva y emocionalmente requiere de parte de las personas conocimientos, aclaración de sus mitos, prejuicios y tabúes, aclaración de sus pensamientos y de sus sentimientos al expresar su sexualidad en la convivencia humana. Requiere también que estas personas enfrenten sincera y abiertamente su sexualidad en un contexto histórico, que exige diálogo, mayor estudio sobre el tema, sinceridad y honestidad, dentro de un marco ético de derechos humanos que permita la toma de decisiones responsablemente, sin miedos, sin dudas y sin manipulaciones de ninguna clase. Se asume que los seres humanos 
que participan consciente y sistemáticamente en el análisis de su propia realidad biopsicosocial son los que pueden transformarla.

La sexualidad es una dimensión de la personalidad, impregnada desde la concepción por las mismas características biopsicosociales y espirituales de la persona. Nace, evoluciona con ella y se expresa en la relación de la persona consigo misma y en la convivencia con los otros seres humanos a través de los vínculos emocionales que se establezcan, del papel sexual que se nos asigna socialmente; a través de la respuesta sexual y el erotismo que se vivan y del ejercicio voluntario o no de la fertilidad.

Las estructuras anatómicas y el funcionamiento fisiológico del sistema endocrino, del sistema nervioso, de los órganos genitales y las zonas erógenas del cuerpo humano se retroalimentan unas a otras para formar la base biológica de la expresión sexual humana. La armonía con que funcionan normalmente estas estructuras, fortalece la organización biológica de la reproducción, del placer y el erotismo, de la respuesta sexual, de los vínculos emocionales entre los seres humanos y del papel sexual.

Las habilidades cognitivas y emocionales de la persona, su organización y funcionamiento biológico, interaccionan desde la concepción con el ambiente sociocultural en el cual vive y le permiten construir patrones de comportamiento único para expresar su sexualidad en cada fase de su desarrollo, en cada época histórica y en cada sociedad en particular.

Un proceso complejo del ser humano es el logro de su identidad sexual, la cual es el aspecto psicológico de la sexualidad y comprende tres elementos indivisibles: la identidad de género, el papel sexual y la orientación sexual. La identidad de género es el sentirse hombre o mujer y manifestarlo externamente a través del papel sexual que se construye en la sociedad en que se vive. La orientación sexual se caracteriza por la preferencia o gusto de la persona para elegir compañero o compañera sexual (Monroy, Aguilar y Morales, s.f.). Esta conceptualización de la sexualidad, entretejida con la vida de la persona, con su entorno sociocultural, participando de un compromiso universal, es humana y supera los límites del determinismo biológico.

La forma en que la persona expresa su sexualidad al relacionarse con las otras personas refleja su concepto de ser humano y su filosofía de la vida. La sexualidad humana, cuando 
se le mira superficialmente puede dar la impresión de ser muy "biológica", pero a medida que se profundiza en su estudio, se torna en una de las facetas más difíciles y conflictivas de la persona, tanto en su aspecto teórico y abstracto, como en sus realizaciones concretas. La dificultad radica en que la sexualidad es personal al tiempo que forma parte de una cultura y de situaciones cambiantes con las circunstancias concretas de cada persona. Es parte de un mundo interior que no se aviene fácilmente a imposiciones venidas desde afuera. Para intentar entender la expresión de la sexualidad humana se debe situar a la persona en su medio cultural y en su momento histórico, pues todo lo que modifique el ambiente modifica la proyección de la sexualidad y a la inversa, el comportamiento sexual de la persona modifica su medio. La sexualidad humana es cambiante, se enfrenta al medio y lo trasciende, tiene historia y su futuro es humano. (Sahagún, 2003)

Son las personas adultas significativas para el niño, para la niña y para el y la adolescente las que con formación e información siempre actualizada, acortan la brecha generacional al emplear un lenguaje común y al expresar su propia sexualidad, sin romper su egosintonía, voluntariamente y con responsabilidad médica, dentro del marco de los derechos humanos. En otras palabras, al asumir su sexualidad las personas adultas logran trascender a las nuevas generaciones con actitudes y conocimientos sanos al educarlos y educarlas.

\section{Aportes para una educación de la expresión de la sexualidad y de la inteligencia emocional}

\subsection{Principios fundamentales}

Construir ambientes de aprendizaje y enseñanza para educar la expresión de la sexualidad continua y cotidianamente, enfatizando la trascendencia del papel que juegan las habilidades de la inteligencia emocional en el proceso, es desafiante y placentero. Dentro del amplio abanico de habilidades que se deben educar en niños, niñas y adolescentes están:

- Las habilidades propias de la inteligencia emocional.

- El respeto a su cuerpo con un claro esquema corporal y con una clara imagen corporal.

- La habilidad de sentirse capaz de encontrarle sentido a la vida.

- La habilidad de construir significativamente a la resolución de problemas. 
- La habilidad de convivir con las demás personas, en cooperación y negociando sus diferencias para sentir así que puede influir en lo que le pase, de que son responsables de su vida.

- La habilidad de reconocer y defender sus derechos.

- La habilidad de cumplir con sus responsabilidades y rendir cuentas.

Desde la concepción, el desarrollo del cerebro humano es producto de un delicado equilibrio entre genes - herencia - y experiencias - ambiente -. Cada contacto corporal, cada movimiento y cada emoción se convierten en una actividad eléctrica y química que propicia la configuración del cerebro, cuyo tamaño y complejidad le permite a la persona adaptarse a su entorno. El estímulo temprano y oportuno al niño y a la niña en el útero repercuten desde ya en desarrollo de las conexiones cerebrales que se activan y responden formando redes sinápticas. Se sabe que el cerebro humano es maleable y que tiene capacidad de reorganización durante toda la vida, cuando estas conexiones se potencian mediante experiencia. Sin embargo hay consenso entre los estudiosos de que es durante la primera infancia cuando el cerebro humano se forma a una velocidad que nunca volverá a repetirse (UNICEF, 2001).

Las enseñanzas y los aprendizajes de conocimientos, de actitudes, de sentimientos y de pensamientos modifican el cerebro para la expresión de la sexualidad inteligente cognitiva y emocionalmente. Entre el intelecto y la emoción existen estrechos vínculos emocionales. Es por tanto una realidad, que la educación sistemática y consciente, es la que provoca los cambios neurobiológicos necesarios para mejorar los talentos o habilidades del ser humano. Es la inteligencia cognitiva mediante los elementos que desarrollan su proceso de funcionamiento, la que permite ser consciente de las emociones: percibirlas, identificarlas, comprenderlas, manipularlas y expresarlas de forma pertinente, en función del lugar, momento y personas implicadas, asumiendo así dominio de ellas.

Gardner, Kornhaber y Wake (2000) señalan que el estudio de las bases biológicas de la inteligencia se encuentra en sus etapas iniciales y aunque es precipitado hacer conclusiones, existen evidencias que sugieren que en las madres embarazadas que sufren tensiones y presiones poco comunes, su niño o niña puede desarrollar cerebros organizados de una forma diferente de los cerebros de los niños y niñas de madres más estables emocionalmente. Sin embargo, los científicos sociales y los historiadores piensan que son 
las bases biológicas del comportamiento humano en sus transacciones con el ambiente las que propician un desarrollo humano armonioso o no.

En el libro "Emociones destructivas", Daniel Goleman (2003), presenta los siguientes aportes del Doctor Mark Greenberg, psicólogo evolutivo e infantil e investigador de los fundamentos neurobiológicos del desarrollo emocional:

- Los efectos provocados por la privación emocional y social en el cerebro infantil pueden influir sobre su desarrollo y su plasticidad. El cerebro es el órgano que más tarda en alcanzar su madurez anatómica y sus conexiones neuronales se establecen en función de las experiencias y del aprendizaje, lo que convierte a los primeros años de vida en una verdadera oportunidad para educar las habilidades cognitivas y emocionales del ser humano.

- Las emociones son señales muy importantes y hay que proporcionarles a los niños, a las niñas y a los y las adolescentes espacios para que tomen conciencia de ellas, verbalicen sus sentimientos y las reconozcan en otras personas. Para aprender este lenguaje emocional es fundamental la educación. La educación produce el florecimiento humano y le permite a la persona entrar en contacto consigo misma, con sus sentimientos y emociones y a la vez ir fortaleciendo las habilidades de la inteligencia emocional.

- Es más sencillo enseñar a niños y a niñas todas las habilidades de la inteligencia emocional durante el periodo en que está conformándose su sistema de circuitos neuronales, que tratar de modificarlos luego.

El reto mayor al que se enfrenta la humanidad consiste en aprender a equilibrar adecuadamente el corazón y la mente. Los estrechos vínculos emocionales que existen entre el intelecto y la emoción llevan a afirmar que la educación transforma el funcionamiento del cerebro y para responder a estas afirmaciones científicas, se crea el programa PATHS (Promoting Alternative Thinking Strategies). El objetivo del mismo es educar para prevenir a nivel primario y proteger así a los niños y a las niñas, mediante la enseñanza de habilidades emocionales esenciales para la vida. El programa forma parte de un movimiento de 
aprendizaje emocional y social que aspira a ser implantado en todas las escuelas, según Mark Greenberg (citado por Goleman, 2003).

Estos programas de educación emocional, son pertinentes y oportunos para iniciar sistemáticamente la educación de la sexualidad humana integrada a ellos. En la educación de la expresión de la sexualidad de niños, de niñas y de adolescentes es fundamental el aprender a valorar en forma adecuada los conocimientos y los sentimientos que se producen tanto por estímulos internos como externos y aprender a manejar la información que se adquiere. Así mismo es importante educarlos y educarlas para que acepten sus propios sentimientos y para que disciernan cuales comportamientos son adecuados para expresarlos. Mediante estrategias concretas y con la mediación de las personas adultas significativas como modelos, los niños, las niñas y los y las adolescentes al interactuar aprenden a valorar la emoción, a sentir y pensar antes de actuar, aspectos claves para una convivencia armoniosa consigo mismo y con los demás seres humanos. Aprenderán además a romper los mitos, prejuicios y tabúes que impregnan la expresión de la sexualidad humana al adquirir conocimientos actualizados y pertinentes.

\subsection{Relaciones humanas}

El amor es lo que hace que las relaciones interpersonales sean verdaderamente humanas y los vínculos emocionales que se establecen con seres humanos significativos son los que permiten a los niños, a las niñas y a los y las adolescentes educar la expresión de su sexualidad en forma inteligente cognitiva y emocionalmente. Para fortalecer este ambiente humano y humanizador, se debe emplear en la relación interpersonal de convivencia las siguientes actitudes básicas que fortalece la educación de la inteligencia emocional y la expresión de la sexualidad de los seres humanos (Ginott, 1972, Bailey, 2001, Goleman, 2001):

- Promover siempre el respeto a los derechos humanos.

- Relacionarse siempre con expectativas realistas.

- Reconocer las características únicas de cada ser humano.

- Aceptar positiva e incondicionalmente a cada persona sin aprobar necesariamente todo lo que hace.

- Comprender a cada ser humano empáticamente, poniéndose en su lugar sin dejar de ser uno mismo. 
- Responder en la relación interpersonal en forma refleja y descriptiva, cada vez que se pueda.

- Corregir atacando la situación y no la persona.

- Darle a cada persona tiempo de reacción.

- Expresar las emociones y los sentimientos y así alfabetizar emocionalmente.

- Ofrecer a cada persona la oportunidad de corregir cada vez que se considere su comportamiento inconveniente para ellas mismas y para las otras personas.

- Permitirle a las personas que cooperen.

Las relaciones humanas perneadas por el amor están fortalecidas por la inteligencia espiritual de las personas y son las habilidades espirituales las que contribuyen a que cada una de ellas le encuentre sentido a la vida. Una orientación espiritual sana contribuye a mejorar la vida del ser humano y fomenta el crecimiento de su capacidad de amar y de desarrollarse. Para Federico Mayor (1997), nada puede sustituir al contacto humano y el caudal de experiencias que transmite.

Hellen (1990) considera que el desarrollo espiritual es un proceso natural que ocurre espontáneamente en las personas cuando encuentran apoyo y las circunstancias apropiadas.

En este momento histórico las habilidades espirituales incentivan la reflexión y la acción del ser humano para encontrar razones para vivir consigo mismo y con todos los seres del universo. La fe, afirma Froom (1978), juega un papel muy importante dentro de las habilidades espirituales. Sin fe la persona se vuelve estéril, pierde toda esperanza y le teme a la esencia misma de su ser.

Las habilidades espirituales se respaldan, por lo general en una religión. La religión ha proporcionado al ser humano, durante miles de años, razones para vivir cuando la existencia se hace difícil y le ha dado coraje para dominar las fuerzas hostiles de la naturaleza indomable. La religión proporciona pautas y sistemas de valores para que los seres humanos puedan convivir en armonía (Jowrard y Landsman, 1987). 
Así la religión es una forma de ser y su orientación es saludable si mejora la calidad de vida y fomenta el desarrollo de uno mismo como ser humano, su capacidad de amar, de ser productivo y creativo en la convivencia con los demás.

La fe en creer es tan racional o tan irracional como la fe en no creer.

Creer o no creer son dos formas de fe cuyos fundamentos pueden ser más o menos valorados, pero ambas posturas, quiérase o no se traducen en actuaciones concretas... Creer en un Creador y en un destino trascendente del espíritu, implica respetarse a sí mismo, respetar la propia vida y la propia integridad, así como respetar igualmente la de los demás seres humanos. (Sahagún, 2003, p. 165)

Una educación de la expresión de la sexualidad inteligente cognitiva y emocionalmente lleva a la persona a convivir con los demás sin dejar de ser ella misma. Y es que, la humanidad es cada vez más consciente de los derechos de los seres humanos, sin distinción de sexo y lucha por que cada quien asuma la responsabilidad de sus actos en su vida privada y pública, de acuerdo con su propia filosofía de vida y su escala de valores.

Ginott, citado por Faber y Mazlish (1982), insiste en que los niños, las niñas y los y las adolescentes son personas y que todos sus sentimientos están permitidos aunque sus acciones se limitan. Por tanto, en las relaciones interpersonales se tiene que dar espacio para comunicarnos nuestros sentimientos y reflejarlos como en un espejo, con una imagen positiva y realista que logre proyectar:

- Que un sentimiento es un hecho

- Que dos o más sentimientos contradictorios pueden existir simultáneamente,

- Que los sentimientos de cada ser humano son únicos, y

- Que cuando se identifican y se aceptan los sentimientos, las personas entran en mayor contacto con aquello que sienten.

\section{A manera de conclusión}

La educación de la expresión de sexualidad comienza desde antes de la concepción misma del nuevo ser, desde el momento en que la pareja decide que quiere o no ser papá y mamá. La maternidad y la paternidad deben fundarse en decisiones voluntarias y responsables. 
El niño y la niña en edad preescolar necesitan aprender sobre expresión de sexualidad en forma natural. En esta edad los patrones de convivencia familiar orientan la mayoría de sus formas de comportamiento. El hogar se convierte en el primer laboratorio de educación de expresión de la sexualidad, donde el niño y la niña aprenden cómo un hombre y una mujer conviven. Las personas adultas consciente o inconscientemente son ejemplos vivientes con sus actos cotidianos y por lo tanto, les corresponde clarificar y fortalecer sus actitudes, valores y conocimientos de su propia expresión de la sexualidad para sistematizar conscientemente una serie de acciones para la educación de la expresión de sexualidad y la inteligencia emocional de niños y niñas.

Dentro de estos procesos de enseñanza y aprendizaje de la expresión de la sexualidad, en edad preescolar, los actos dicen más que las palabras. El lenguaje es esencial para que el pequeño y la pequeña vayan construyendo su esquema corporal, dando a cada parte del cuerpo el nombre correcto; dentro de este vocabulario hay que incluir los nombres exactos de los genitales femeninos y masculinos. Así mismo, las preguntas de los niños y las niñas sobre sexualidad se tienen que responder en forma clara y sencilla, con respuestas centradas en los intereses y necesidades de estas personitas, de tal forma que el diálogo quede abierto para retomarse en cualquier momento pertinente. Preguntar y repreguntar, van a permitir al niño y a la niña construir conceptos y acciones en torno a la expresión de sexualidad y fortalecer sus habilidades, propias de la inteligencia emocional.

A partir de los 7 años, más o menos, el niño y la niña tienen una noción de la permanencia de su sexo y del papel femenino o masculino aceptado por la sociedad en que viven, factores muy importantes para el desarrollo del concepto de sí mismo y de sí misma.

El desarrollo del pensamiento lógico de este niño y de esta niña, les permiten hacer comparaciones entre la forma que las personas adultas se relacionan, los papeles sexuales que asumen y las formas en que se autocontrolan al resolver conflictos. Además, a partir de los 7 años, mediante el juego y el trabajo en grupo, el niño y la niña aprenden a fortalecer sus habilidades para expresar sentimientos, tomar y expresar una posición personal, aunque en algunas ocasiones les cueste reconstruirla, a partir de los aportes del otro u otra. Así mismo pueden seguir instrucciones y aceptar las normas que se establecen, habilidades que fortalecen su inteligencia emocional y que contribuyen a que se sientan fuertes y seguros, capaces de convivir en armonía, respetando sus derechos y los de los demás. 
La persona adulta, con conocimiento de cada niño y niña, aceptando la diversidad de diferencias individuales, buscan la forma de incentivar procesos para la adquisición de conocimientos y la aclaración de mitos, prejuicios y tabúes, con miras a que , sin imponer sus puntos de vista, cada persona construya una expresión de la sexualidad inteligente cognitiva y emocionalmente.

Asumir la educación de la expresión de la sexualidad de adolescentes, es quizá el reto mayor que asumen las personas adultas, deseosas de que las nuevas generaciones disfruten de una expresión de la sexualidad armoniosa, respetuosa y responsable. Ante los rápidos cambios de la época, es una labor no de un instante, sino de toda la vida, que se ajusta al desarrollo del adolescente y de la adolescente. No es ético despojar a los adolescentes y las adolescentes de sus derechos y obligaciones. Por este motivo es conveniente que estas personas se eduquen en un ambiente democrático, donde con toda libertad expresen sus puntos de vista, sus dudas y cometarios en torno a la expresión de la sexualidad. Hoy día el adolescente y la adolescente, por un lado disponen de mayores oportunidades de intimidad, y por otro, son bombardeados por una gran cantidad de estímulos erotizantes que promueven el riesgo de vivir una expresión de la sexualidad que rompa con su opción de construir y reconstruir su existencia voluntariamente.

Con base en los planteamientos anteriores, es pertinente que las personas adultas abran espacios para que grupos de niños, niñas y adolescentes, discutan en su compañía, dilemas sexuales de interés para ellos y ellas, que les permitan expresar sus opiniones y construir y reconstruir sus posiciones, así como expresarlas y defenderlas públicamente. Al dialogar y discutir él y ella van fortaleciendo la construcción de sus conocimientos, de su escala de valores y de su filosofía de la vida, fundamento que respalda la expresión de su sexualidad en la convivencia cotidiana y que fortalece sus habilidades emocionales.

Esta educación así entendida, humaniza y permite durante toda la vida un florecimiento de la persona, de su conocimiento, de sus actitudes, de su facultad de juicio y acción para responder de manera flexible y diversa a los retos de mundo en el tiempo y en el espacio. La educación encierra un tesoro y sus alcances llevan a aprender a vivir juntos, a aprender a conocer, a aprender a hacer y a aprender a ser. (Delors 1996) 


\section{Referencias}

Bailey, Becky. (2001). Edúquelos con amor. México: Pearson Educación.

Carballo, Sonia. (1998). Intervenciones para ayudar a construir la inteligencia emocional. Educación, 22 (1), 77-90. San José. Costa Rica.

Carballo, Sonia. (2002). Educación de la expresión de la sexualidad humana. Educación, 26 (1), 29-46. San José. Costa Rica.

Coleman, Paul. (2001). ¿Cómo decirlo? A los niños. Consuele a sus hijos, resuelva sus problemas y transmítales valores. México: Pearson Educación.

Delors, Jacques. (1996). La educación encierra un tesoro. Madrid: Ediciones UNESCO.

Faber, Adele y Mazlish, Elaine. (1982). Padres liberados, hijos liberados. México: Editorial Diana.

Fromm, Erik. (1978). Tener o ser. México: Fondo de Cultura Económica.

Gardner, Howard. (1993). La mente no escolarizada. España: Paidós.

Ginott, Haim. (1947). Maestros alumnos. México: PAX.

Goleman, Daniel. (1995). La inteligencia emocional. México: Javier Vergara Editor.

Goleman, Daniel. (2003). Emociones destructivas. Buenos Aires: Vergara Grupo Zeta.

Hellen, David. (1990). ¿Cómo hablarle a su hijo de Dios? Colombia: Norma.

Jowrard, Sidney y Landsman, Ted. (1987). La personalidad saludable. México: Trillás.

Gardner, Howard, Kornhaber, Mindy y Wake, Warren (2000). Inteligencia. Múltiples perspectivas. Buenos Aires: Aique Grupo Editor S.A.

Mayor, Federico. (1997). Discurso. Nurcia: Organización de las Naciones Unidas para la Educación, la Ciencia y la Cultura.

Monroy, Anameli; Aguilar, José Ángel; y Morales, Martha (1985). Salud, sexualidad y adolescencia. México: Centro de Orientación para adolescentes, A.C.

Sahagún, Alberto. (2003). Integración sexual humana. México: Trillás.

Shapiro, Laurence. (1997). La inteligencia emocional de los niños. Buenos Aires: Javier Vergara Editor.

UNICEF. (2001). Estado Mundial de la Infancia. Nueva York: autor. 